\title{
THE FAMILY FORMULA FOR LEPTONS AND QUARKS
}

\author{
NIKOLA PERKOVIC \\ Institute of Physics and Mathematics, Faculty of Science, University of \\ Novi Sad, Dositej Obradovic square 3, Novi Sad, 21000, Serbia
}

\begin{abstract}
The problem of Yukawa couplings being arbitrary parameters in the Standard Model Higgs mechanism is a long standing one due to their formulaic dependence on the Higgs Vacuum Expectation Value. We will attempt to solve this problem and provide a strong argument that the Yukawa couplings of charged leptons and down type quarks are not arbitrary parameters in the SM. A new methodology for predicting the Yukawa couplings will be presented by using Compton wavelengths, the Rydberg Constant and g-factors of charged leptons instead of relying on the Higgs VEV. We will then proceed to rewrite this new method in terms of an empirical formula that depends on the running of the fine-structure constant on the Q scale, charge and lepton quantum numbers and g-factors to predict the values of the Yukawa couplings for all three generations of charged leptons and d-type quarks. We will also touch on the subject of neutrinos both as Majorana and Dirac fermions respectively and make a prediction for the lightest possible Majorana neutrino and the differences between Dirac neutrinos and anti-neutrinos. We conclude that the Yukawa couplings are not arbitrary parameters in the SM and that this new formula provides very accurate results.
\end{abstract}

Keywords: Higgs Mechanism, Yukawa Coupling, Fine structure constant, Leptons, Quarks

PACS Nos: 12.15.Ff, 12.15.Hh, 12.15.Lk

\section{Introduction}

In the Standard Model of Particle Physics ${ }^{1-4}$, electroweak symmetry breaking ${ }^{5-8}$ is responsible for the mass generation of $\mathrm{W}$ and $\mathrm{Z}$ gauge bosons thus rendering the weak interactions short ranged. The Standard Model scalar potential is:

$$
\mathrm{V}(\Phi)=\mathrm{m}^{2} \Phi^{\dagger} \Phi+\lambda\left(\Phi^{\dagger} \Phi\right)^{2}
$$

where the Higgs field $\Phi$ is a self-interacting $\mathrm{SU}(2)_{\mathrm{L}}$ complex doublet that has four real degrees of freedom, with weak hypercharge $\mathrm{Y}=1$ and $\mathrm{V}(\Phi)$ is the most general renormalizable scalar potential and if the quadratic term is negative the neutral component of the scalar doublet acquires a non-zero vacuum expectation value $v=\left(\sqrt{2} \mathrm{G}_{\mathrm{F}}\right)^{-1 / 2}$ which is approximately $246,22 \mathrm{GeV}$. We should also point out that 


$$
\Phi=\frac{1}{\sqrt{2}}\left(\begin{array}{c}
\sqrt{2 \phi^{+}} \\
\phi^{0}+i a^{0}
\end{array}\right)
$$

where $\phi^{0}$ and $a^{0}$ are the $\mathrm{CP}$-even and $\mathrm{CP}$-odd neutral components, and $\phi^{+}$is the complex charged component of the Higgs doublet, respectively. The global minimum of the theory defines the ground state, and spontaneous symmetry breaking implies that there is a symmetry of the system that is not respected by the ground state. From the four generators of the $\mathrm{SU}(2)_{L} \times \mathrm{U}(1)_{Y}$ gauge group, three are spontaneously broken, implying that they lead to non-trivial transformations of the ground state and indicate the existence of three massless Goldstone bosons identified with three of the four Higgs field degrees of freedom. The Higgs field couples to the $W_{\mu}$ and $B_{\mu}$ gauge fields associated with the $\mathrm{SU}(2)_{L} \times \mathrm{U}(1)_{Y}$ local symmetry through the covariant derivative appearing in the kinetic term of the Higgs Lagrangian

$$
\mathcal{L}_{\text {Higgs }}=\left(D_{\mu} \Phi\right)^{\dagger}\left(D^{\mu} \Phi\right)-V(\Phi)
$$

Where the covariant derivative equals

$$
\mathrm{D}_{\mu}=\partial_{\mu}+\frac{i g \sigma^{a} \mathrm{~W}_{\mu}^{a}}{2}+\frac{i \mathrm{~g}^{\prime} \mathrm{YB}_{\mu}}{2}
$$

$\mathrm{g}$ and $\mathrm{g}^{\prime}$ are the $\mathrm{SU}(2)$ and $\mathrm{U}(1)$ gauge couplings, respectively, and $\sigma^{a}$ where $a=1,2,3$ are the typical Pauli matrices. As a result, the neutral and the two charged massless Goldstone degrees of freedom mix with the gauge fields corresponding to the broken generators of $S U(2)_{L} \times U(1)_{Y}$ and become the longitudinal components of the $\mathrm{Z}$ and $\mathrm{W}$ gauge bosons, respectively. The $\mathrm{Z}$ and $\mathrm{W}$ gauge bosons acquire masses $\mathrm{M}_{\mathrm{W}}=\mathrm{g} v / 2$ and $\mathrm{M}_{\mathrm{Z}}=\left(\mathrm{g}^{\prime}+\mathrm{g}\right) v / 2$. The fourth generator remains unbroken sinc $\overline{\text { it }}$ is the one associated to the conserved $\mathrm{U}(1)_{\mathrm{QED}}$ gauge symmetry therefore its corresponding gauge field remains massless or in other words, the photon is massless. Similarly the eight color gauge bosons, the gluons, corresponding to the conserved $\mathrm{SU}(3)_{C}$ gauge symmetry with eight unbroken generators, also remain massless. Therefore, from the initial four degrees of freedom of the Higgs field, two are absorbed by the $\mathrm{W}^{ \pm}$gauge bosons, one by the $\mathrm{Z}^{0}$ gauge boson, and there is one remaining degree of freedom $\mathrm{H}$, that is the physical Higgs boson. The Higgs boson is neutral under the electromagnetic interactions and transforms as a singlet under $\mathrm{SU}(3)_{C}$ and hence does not couple at tree level to the massless photons and gluons. The mass of the Higgs boson ${ }^{9}$ is given as $\mathrm{m}_{\mathrm{h}}=\sqrt{2 \lambda v^{2}}$, where $\lambda$ is a free coupling parameter and therefore the mass of the Higgs boson is not predicted in the Standard Model. With the Higgs field in the unitary gauge, the $\mathrm{SU}(2)_{L} \times \mathrm{U}(1)_{Y}$ invariant Yukawa Lagrangian For leptons takes the form:

$$
\mathcal{L}_{f}=-\lambda_{f}\left(\overline{\mathrm{L}} \Phi \mathrm{e}_{\mathrm{R}}+\Phi^{\dagger} \overline{\mathrm{e}}_{\mathrm{R}} \mathrm{L}\right)=-\frac{\lambda_{f} v}{\sqrt{2}} \overline{\mathrm{e}} \mathrm{e}-\frac{\lambda_{f} h}{\sqrt{2}} \overline{\mathrm{e}} \mathrm{e}
$$

The respective masses of fermions are not predicted since the Yukawa coupling $\lambda_{f}$ is a free parameter provided in the formula:

$$
\mathrm{m}_{f}=\frac{\lambda_{f} v}{\sqrt{2}}
$$


in that sense the Higgs mechanism does not predict any of the fermion masses. The results from eq. (6) are listed in the table below.

Table 1: Flavors, masses and numerical values of charged lepton Yukawa couplings

\begin{tabular}{clc}
\hline$f$ & $\operatorname{mass}\left[\mathrm{MeV} \cdot \mathrm{c}^{-2}\right]$ & $\lambda_{f}$ \\
\hline$e$ & $0.5109989461(31)$ & $2.935(17) \times 10^{-6}$ \\
$\mu$ & $105.6583745(24)$ & $6,07(10) \times 10^{-4}$ \\
$\tau$ & $1776.82(16)$ & $1.02(04) \times 10^{-2}$ \\
\hline
\end{tabular}

It is possible to estimate the strength of the fermion-fermion-Higgs interactions

$$
\mathcal{L}_{f f h}=\frac{\mathrm{m}_{\mathrm{e}}}{v} \overline{\mathrm{e}} \mathrm{e} h-\frac{\mathrm{m}_{\mathrm{u}}}{v} \overline{\mathrm{u}} \mathrm{u} h+\cdots
$$

where $\mathrm{m}_{\mathrm{e}}$ is the mass of an electron and $\mathrm{m}_{\mathrm{u}}$ is the mass of an up quark. A very important consequence of the fermion-fermion-Higgs interaction is its direct dependence on fermion masses. The larger the mass the stronger this interaction becomes. In order to make sure that Yukawa couplings are no longer arbitrary parameters in the SM Higgs mechanism, we have to avoid using the Higgs VEV. Therefore we rewrite the formula in eq. (6) as:

$$
\lambda_{l}=\frac{\left(\lambda_{\mathrm{C}, l} \cdot \mathrm{R}_{\infty} \cdot \mathrm{n}_{\mathrm{g}}^{\mathrm{L}_{f} \mathrm{~g}_{f}}\right)^{\mathrm{n}_{\mathrm{g}}^{1-\frac{\mathrm{Q}^{2}}{\mathrm{~L}^{2}} \mathrm{~N}}}}{\mathrm{~N}^{2}}
$$

Where $\lambda_{l}$ is the lepton Yukawa coupling, $l=e, \mu, \tau$ are the flavors for electrons as the first generation, muons as second and tau leptons as third respectively, $\lambda_{\mathrm{C}, l}$ is the Compton wavelength of the lepton, $\mathrm{g}_{f}$ is the $\mathrm{g}$-factor of the charged lepton, $\mathrm{L}_{f}=\mathrm{L}_{e}, \mathrm{~L}_{\mu}, \mathrm{L}_{\tau}$ is the lepton family number, $\mathrm{n}_{\mathrm{g}} \equiv 3$ is the number of families/generations, $\mathrm{R}_{\infty}$ is the Rydberg constant and $\mathrm{N}$ is a quantum number that equals $\mathrm{N}=i^{\mathrm{Q}^{2} / \mathrm{L}^{2}}$ where $i=1,2,3$ represents the mass eigenstates, $\mathrm{Q}$ and $\mathrm{L}$ are the electromagnetic charge ${ }^{1}$ quantum number and Lepton number, respectively. The charge quantum number equals

$$
\mathrm{Q}=\mathrm{T}_{3}+\frac{1}{2} \mathrm{Y}
$$

where $\mathrm{T}_{3}$ is the third component of weak isospin and $\mathrm{Y}$ is the weak hypercharge. The lepton number equals $\mathrm{L}=\mathrm{n}_{l}-\mathrm{n}_{\bar{l}}$ where $\mathrm{n}_{l}$ represents the number of leptons and $\mathrm{n}_{\bar{l}}$ the number of anti-leptons. Compton wavelengths of electrons, muons and tau leptons are known to high accuracy however the Rydberg constant and the g-factors of electrons and muons are some of the best measured quantities in all of physics, along with the fine structure constant $\alpha$ which will be used in the more complex version of the formula from eq. (8), replacing the Rydberg constant and Compton wavelengths. Eq. (8) provides approximately the same values as does eq. (6), the values of which are provided in Table 1. The problem we are facing now is that we rely on Compton wavelengths that are proportional to a particle's mass and the Rydberg constant meaning that we cannot predict the Yukawa couplings of either leptons or quarks until we remove $\lambda_{C, l}$ and $\mathrm{R}_{\infty}$ from the formula in eq. (8).

\footnotetext{
${ }^{1}$ The electromagnetic charge quantum number can also be represented as Q/e where e denotes the elementary charge.
} 


\section{The Charged Lepton Family Formula}

Following the relationship the Rydberg constant has with the Compton wavelength $R_{\infty}=\alpha^{2} / 2 \lambda_{C, \tau}$ we can rewrite the formula from eq. (8), however this only applies for the Compton wavelength of electrons, not muons and tau leptons. The revised formula from eq. (8) takes the form in which we only rely on the fine structure constant, the g-factor and the respective quantum numbers. We rewrite the formula in integral form both to eliminate the Compton wavelengths and the Rydberg constant as previously mentioned as well as to describe the relationship between the Yukawa couplings, the fine structure constant and the Higgs field. The formula is:

$$
\lambda_{l}=\left(\frac{\mathrm{n}_{\mathrm{g}}^{\mathrm{L}_{\mathrm{f}} \mathrm{g}_{\mathrm{f}}}}{(1+\Delta \mathrm{q})} \int_{0}^{\alpha(\mathrm{Q})} \Phi^{\mathrm{N}} d \Phi\right)^{1-\frac{\mathrm{Q}^{2}}{\mathrm{~L}^{2} \mathrm{~N}}} / \mathrm{N}^{2}
$$

where $\alpha(Q)$ is the running value of the fine structure constant on the scale $Q, \Phi$ is the Higgs field and $\Delta \mathrm{q}$ encapsulates the higher order QED corrections and can be expressed as a power series expansion in the renormalized electromagnetic coupling constant $\alpha \Delta \mathrm{q}=\sum_{i=0}^{\infty} \Delta \mathrm{q}^{j}$ in which the index $j$ gives the power of $\alpha$ that appears in $\Delta \mathrm{q}^{j}$. The formula can thus be further simplified to be:

$$
\lambda_{l}=\left(\frac{\alpha^{\mathrm{N}+1}(\mathrm{Q}) \cdot \mathrm{n}_{\mathrm{g}}^{\mathrm{L}_{f} \mathrm{~g}_{f}}}{(\mathrm{~N}+1)(1+\Delta \mathrm{q})}\right)^{\substack{1-\frac{\mathrm{Q}^{2}}{\mathrm{~L}^{2}} \\ \mathrm{n}_{\mathrm{N}}}} / \mathrm{N}^{2}
$$

When $i=1$ and therefore $l=e, \mathrm{Q}=-1, \mathrm{~L}=1, \mathrm{~L}_{f}=\mathrm{L}_{e}=1$ we obtain the formula for the electron Yukawa coupling

$$
\lambda_{e}=\frac{\alpha^{2} \cdot 3^{g_{f}}}{2}
$$

When $i=2$ and therefore $l=\mu, \mathrm{Q}=-1, \mathrm{~L}=1, \mathrm{~L}_{f}=\mathrm{L}_{\mu}=1$ we obtain the formula for the muon Yukawa coupling

$$
\lambda_{\mu}=\left(\frac{\alpha^{3}\left(\mathrm{M}_{\mu}\right) \cdot 3^{\mathrm{g}_{f}}}{3 c_{3, \mu}^{-1}}\right)^{1 / 3} / 4
$$

where $\alpha\left(\mathrm{M}_{\mu}\right)$ is the value of the fine structure constant on the muonic scale, that is when $\mathrm{Q}=\mathrm{M}_{\mu}$. We will use the $\overline{\mathrm{MS}}$ renormalization scheme. The effective value of the fine structure constant is obtained by using the equation ${ }^{10}$ :

$$
\alpha(Q)=\frac{\alpha}{1-\widehat{\Pi}(Q)}
$$

where $\widehat{\Pi}(\mathrm{Q})$ is the photon vacuum polarization function which can be written as $\widehat{\Pi}(\mathrm{Q})=\sum_{i=1}^{\infty} \widehat{\Pi}^{i}(\mathrm{Q})$ where each term receives contributions from all fermion flavors. In the $\overline{\mathrm{MS}}$ renormalization scheme 
the counter terms are chosen so that they only contain divergent pieces with the addition of certain constants. One-loop counter terms are proportional to $\Delta=1 / \varepsilon-\gamma_{\mathrm{E}}+\ln (4 \pi)+O(\varepsilon)$ where $\gamma_{\mathrm{E}}$ is the Euler-Mascheroni constant. An appropriate choice for the 't Hooft mass is $\mu=\mathrm{M}_{\mu}$ and therefore we write $\alpha(\mathrm{Q})=\alpha\left(\mathrm{M}_{\mu}\right)$. Ultimately we get the equation:

$$
\alpha\left(M_{\mu}\right)=\frac{\alpha}{1-\frac{\alpha}{3 \pi} \ln \left(\frac{m_{\mu}^{2}}{m_{e}^{2}}\right)}+\frac{\alpha^{2}}{4 \pi^{2}} \ln \left(\frac{m_{\mu}^{2}}{m_{e}^{2}}\right)
$$

The equation above provides a value that is listed in Table 2. The electroweak radiative corrections for muons

$$
(1+\Delta \mathrm{q})=c_{3, \mu}^{-1}=\left\{f\left(\frac{\mathrm{m}_{e}}{\mathrm{~m}_{\mu}}\right)\left[1+\frac{\alpha}{4 \pi} 2\left(\frac{25}{4}-\pi^{2}\right)\right]\right\}^{-1}
$$

where $f$ denotes the phase space factor for one massive particle in the final state. The phase space factor is almost negligible for the muon decay $f\left(\mathrm{~m}_{e} / \mathrm{m}_{\mu}\right)=0.999813$. This provides a value of $c_{3, \mu}^{-1}=1.0044041434(36)$.

When $i=3$ and therefore $l=\tau, \mathrm{Q}=-1, \mathrm{~L}=1, \mathrm{~L}_{f}=\mathrm{L}_{\tau}=1$ we obtain the formula for the tau lepton Yukawa coupling:

$$
\lambda_{\tau}=\left(\frac{\alpha^{4}\left(\mathrm{M}_{\tau}\right) \cdot 3^{\mathrm{g}_{f}}}{4 c_{3, \tau}^{-1}}\right)^{1 / 9} / 9
$$

Equation (17) also requires us to apply the $\overline{\mathrm{MS}}$ renormalization scheme. The first step is the same, as it always is, as for muons in eq. (14) thus we'll skip it. The scale Q now equals the tau lepton mass $Q=$ $\mathrm{M}_{\tau}$ and, as pointed out by A. A. Pivovarov in the paper: "Running electromagnetic coupling constant: low energy normalization and the value at $\mathrm{M}_{\mathrm{Z}}$ " in Ref. 11, the total finite renormalization between the fine structure constant and the $\overline{\mathrm{MS}}$ renormalization scheme is given by $\Delta^{4}\left(\mathrm{M}_{\tau}\right)=\Delta^{l}\left(\mathrm{M}_{\tau}\right)+$ $\Delta^{u d s}\left(\mathrm{M}_{\tau}\right)+\Delta^{c}\left(\mathrm{M}_{\tau}\right)=32.7889$ where $\Delta^{l}\left(\mathrm{M}_{\tau}\right)$ accounts for the lepton contribution, $\Delta^{u d s}\left(\mathrm{M}_{\tau}\right)$ for the contribution of up, down and strange quarks and $\Delta^{c}\left(\mathrm{M}_{\tau}\right)$ accounts for the charm quark contribution. This leads us to:

$$
\frac{3 \pi}{\alpha\left(M_{\tau}\right)}=\frac{3 \pi}{\alpha}-\Delta^{4}\left(M_{\tau}\right)
$$

Where the result for $\alpha\left(\mathrm{M}_{\tau}\right)$ is provided in Table 2. The electroweak corrections for tau leptons are slightly more complex to calculate than its muonic counterpart but we can simplify the calculations and obtain a value of $(1+\Delta q)=c_{3, \tau}^{-1}=0.1778963(33)$ by using the formula:

$$
\frac{\tau_{\tau}}{\tau_{\mu}}=\frac{\mathrm{m}_{\mu}^{5}}{\mathrm{~m}_{\tau}^{5}} c_{3, \tau}^{-1}
$$


where $\tau_{\tau}=2.906(10) \times 10^{-13} s$ and $\tau_{\mu}=2.1969811(22) \times 10^{-6} s$ are the mean lifetimes of tau and muons respectively. There is an alternative way to calculate tau lepton electroweak corrections ${ }^{12}$, ${ }^{13}$ but it involves the use of CKM matrix parameters $\left|V_{u d}\right|$ and $\left|V_{u s}\right|$ which we will introduce in the following section for quarks.

Table 2: Flavors, masses, g-factors, effective QED coupling values and Yukawa couplings of charged leptons obtained by the formula from eq. (10) and (11) with the predicted value of the tau lepton g-factor.

\begin{tabular}{ccccc}
\hline$l$ & mass $\left[\mathrm{MeVc}^{-2}\right]$ & $\mathrm{g}_{f}$ & $\alpha^{-1}(\mathrm{Q})$ & $\lambda_{l}$ \\
\hline$e$ & $0.5109989461(31)$ & $-2.00231930436182(52)$ & $137.035999139(31)$ & $2.951(19) \times 10^{-6}$ \\
$\mu$ & $105.6583745(24)$ & $-2.0023318418(13)$ & $135.90(09)$ & $6.12(08) \times 10^{-4}$ \\
$\tau$ & $1776.82(16)$ & $-2.002354(42)$ & $133.557(43)$ & $1.06(05) \times 10^{-2}$ \\
\hline
\end{tabular}

The values of Yukawa couplings from Table 2 are in great agreement with the known values from Table 1, within the respective uncertainties.

\section{The Quark Formula}

\subsection{The CKM Matrix}

In the Standard Model, the quark mass eigenstates (physical states) do not take part as pure states in weak interactions. The unitary transformation connecting the two bases of mass and weak eigenstates is represented by the Cabibbo-Kobayashi-Maskawa (CKM) matrix. By convention, the charge $Q=$ $2 / 3$ quarks ( $\mathrm{u}, \mathrm{c}$ and $\mathrm{t}$ ) are chosen to be pure states, and flavour mixing is described in terms of a $3 \times 3$ matrix operating on the $\mathrm{d}, \mathrm{s}$ and $\mathrm{b}$ quark states:

$$
\left(\begin{array}{l}
d^{\prime} \\
s^{\prime} \\
b^{\prime}
\end{array}\right)=V_{C K M}\left(\begin{array}{l}
d \\
s \\
b
\end{array}\right)=\left(\begin{array}{lll}
V_{u d} & V_{u s} & V_{u b} \\
V_{c d} & V_{c s} & V_{c b} \\
V_{t d} & V_{t s} & V_{t b}
\end{array}\right)\left(\begin{array}{l}
d \\
s \\
b
\end{array}\right)
$$

Thus $d^{\prime}, s^{\prime}$ and $b^{\prime}$, instead of $d, s$ and $b$, are partners of $u, c$ and $t$ respectively within the weak isospin doublets ${ }^{14}$ and they describe flavor whereas $d, s$ and $b$ describe mass. The values of CKM parameters are provided ${ }^{15}$ by the Particle Data Group (PDG).

\subsection{The Quark formulae}

Do to the nature of the CKM matrix, the quark formula for Yukawa couplings can only account the dtype quarks, that is the down, strange and bottom quarks. The lepton number $\mathrm{L}$ is replaced by the Baryon number $\mathrm{B}=1 / 3$ for all three flavors which also changes the quantum number $\mathrm{N}$ so that $\mathrm{N}=$ $i^{Q^{2} / B^{2}}$. The lepton family numbers $\mathrm{L}_{e}, \mathrm{~L}_{\mu}, \mathrm{L}_{\tau}$ are replaced with strangeness $\mathrm{S}=-1$ for strange quarks and bottomness $\mathrm{B}^{\prime}=-1$ for bottom quarks for the second and third generation respectively. Down quarks do not have a "downness" therefore in their formula the lepton family number is replaced with the value of zero. One shouldn't make a mistake of using the third component of isospin $I_{3}$ to replace $\mathrm{L}_{e}$. The formula for quarks is: 


$$
\lambda_{q}=\left(\frac{\alpha^{\mathrm{N}+1}(\mathrm{Q}) \cdot \mathrm{n}_{\mathrm{g}}^{\mathrm{Q}_{f} \mathrm{~g}_{f}}}{(\mathrm{~N}+1)(1+\Delta \mathrm{q})}\right)^{\mathrm{n}_{\mathrm{g}}{ }^{1-\frac{\mathrm{Q}^{2}}{\mathrm{~B}^{2}} \mathrm{~N}}} / \mathrm{N}^{2}
$$

When $i=1$ and therefore $q=d, \mathrm{Q}=-1 / 3, \mathrm{~B}=1 / 3, \mathrm{Q}_{f}=0$ the formula for down quarks is $\lambda_{d}=$ $\alpha^{2} / 2$ where we used $\alpha\left(\mathrm{M}_{d}\right) \cong \alpha$ for the sake of simplicity. Having in mind that the down quark is not much more massive than the electron, we can safely ignore the running of the fine structure constant without sacrificing any relevant accuracy.

When $i=2$ and therefore $q=s, \mathrm{Q}=-1 / 3, \mathrm{~B}=1 / 3, \mathrm{Q}_{f}=\mathrm{S}=-1$ the formula for strange quarks is

$$
\lambda_{s}=\left(\frac{\alpha^{3}\left(\mathrm{M}_{s}\right) \cdot 3^{\mathrm{S} \cdot \mathrm{g}_{f}}}{3\left(\left|V_{c s}\right|^{2} c_{3, s}\right)^{-1}}\right)^{1 / 3} / 4
$$

Where the electroweak corrections correspond to a coefficient $c_{3, S}$ obtained from the strange quark mean lifetime and the $\left|V_{c s}\right|$ CKM matrix parameter.

When $i=3$ and therefore $q=b, \mathrm{Q}=-1 / 3, \mathrm{~B}=1 / 3, \mathrm{Q}_{f}=\mathrm{B}^{\prime}=-1$ The formula for bottom quarks is

$$
\lambda_{b}=\left(\frac{\alpha^{4}\left(\mathrm{M}_{b}\right) \cdot 3^{\mathrm{B}^{\prime} \cdot \mathrm{g}_{f}}}{4\left(\left|V_{c b}\right|^{2} c_{3, b}\right)^{-1}}\right)^{1 / 9} / 9
$$

The values for quarks are provided in Table 3. The values of CKM parameters and the coefficients are known from experimental results ${ }^{16,17}$.

Table 3: Flavors, masses, g-factors, effective QED coupling values and Yukawa couplings of d-type quarks obtained by the formula from eq. (10) and (11). The values of g-factors are approximated from the Dirac equation.

\begin{tabular}{ccccc}
\hline$q$ & mass $\left[\mathrm{MeVc}^{-2}\right]$ & $\mathrm{g}_{f}$ & $\alpha^{-1}(\mathrm{Q})$ & $\lambda_{l}$ \\
\hline$d$ & $4.64(24)$ & -2 & $\sim 137$ & $2.663(35)$ \\
& & & & $\times 10^{-5}$ \\
\multirow{2}{*}{$s$} & $94.61(38)$ & -2 & & $5.434(42)$ \\
& & & & $\times 10^{-4}$ \\
\multirow{2}{*}{$b$} & $4300.36(92)$ & -2 & $\sim 132$ & $2.47(44)$ \\
& & & & $\times 10^{-2}$ \\
\hline
\end{tabular}

\section{Neutrino Conjectures}


Since neutrinos are neutral particles, meaning they have a charge $\mathrm{Q}=0$, the quantum number $\mathrm{N}=$ $i^{\mathrm{Q}^{2} / \mathrm{L}^{2}}$ will always equal one. Neutrinos have three generations, corresponding to charged leptons: electron neutrino, muon neutrino and tau neutrino. However, unlike their charged counterparts neutrinos can oscillate between the flavors, which is known as "neutrino oscillation"18, 19. The formula explains that this phenomenon is caused by neutrinos having no electromagnetic charge which allows them to oscillate, this is known as "neutral particle oscillation". The formula for neutrinos is:

$$
\lambda_{v}=\left(\frac{\alpha^{2} \cdot 3^{\mathrm{L}_{f} \cdot \mathrm{g}_{f}}}{2(1+\Delta q)}\right)^{3}
$$

which means that the mass differences between neutrino flavors depend only on their electroweak corrections. There are two different conjectures on the nature of neutrino masses, the Dirac neutrino and the Majorana neutrino. In simplest terms, Dirac fermions are particles that have anti-particles while Majorana fermions ${ }^{20}$ are their own anti-particles. As of the time this paper is written, all known fermions are Dirac fermions by nature.

Majorana fermions have no magnetic dipole moment ${ }^{21}$, this can be achieved in the formula by stating that $\mathrm{g}_{f}=0$. In that case the lightest neutrino flavor, presumably the electron neutrino if neutrinos follow the "normal hierarchy"22, predicted by the formula is $\mathrm{m}_{v}=0.0033(21) \mathrm{eV}$ from a Yukawa coupling $\lambda_{v}=1.89(22) \times 10^{-14}$.

Dirac fermions have a magnetic dipole moment that is proportional to their mass. This means that the neutrino g-factor has to be larger than zero, however since neutrinos have no electromagnetic charge their $\mathrm{g}$-factor has to be below two $0<\mathrm{g}_{f}<2$. If neutrinos are indeed Dirac fermions, the formula does not predict their mass since it is a part of the Standard Model and the SM does not predict neutrino masses. However the formula does predict that if neutrinos have a positive g-factor then antineutrinos must have a negative one, meaning that their magnetic dipole moment would be negative. This is caused by the lepton family number that has a positive value for neutrinos and a negative value for anti-neutrinos.

\section{Conclusions \& Debate}

The formula from eq. (10) and (11) that rely on the fine structure constant provide very accurate values of charged lepton Yukawa couplings without relying on the use of the Higgs VEV. Therefore the Yukawa couplings are no longer arbitrary parameters within the SM Higgs mechanism with an added possibility to expand to possible new physics Beyond the Standard Model. This possibility can be seen in the neutrino formula from eq. (24) where the electroweak corrections are probably connected with the PMNS matrix. However the PMNS matrix is not a part of the SM since neutrino masses are not predicted by the SM Higgs mechanism. We have still managed to make some predictions both for Majorana and Dirac neutrinos, such as the ligthest possible mass of a Majorana neutrino being approximately 0.0033 (21) $\mathrm{eV}$ and that Dirac neutrinos and anti-neutrinos should have opposite signs on $\mathrm{g}$-factors that is their magnetic dipole moments, such as neutrinos having a positive magnetic dipole moments and anti-neutrinos having a negative one or vice-versa. Besides leptons we have also made some significant predictions when it comes to quarks, focusing on d-type quarks since they describe mass terms in the CKM matrix. The CKM matrix is a fundamental part of the SM unlike its leptonic counterpart, the PMNS matrix. Hopefully these new predictions regarding d-type quarks might help us to improve the accuracy of quark mass measurements. 


\section{References}

1. S.L. Glashow, Nucl. Phys. 20, 579 (1961).

2. S. Weinberg, Phys. Rev. Lett. 19, 1264 (1967).

3. A. Salam, Elementary Particle Theory, eds: Svartholm, Almquist and Wiksells, Stockholm, (1968).

4. S. Glashow, J. Iliopoulos, and L. Maiani, Phys. Rev. D2, 1285 (1970).

5. F. Englert and R. Brout, Phys. Rev. Lett. 13, 321 (1964).

6. P. W. Higgs, Phys. Rev. Lett. 13, 508 (1964).

7. P. W. Higgs Phys. Rev. 145, 1156 (1966).

8. G.S. Guralnik, C.R. Hagen, and T.W. Kibble, Phys. Rev. Lett. 13, 585 (1964).

9. M. Tanabashi et al. (Particle Data Group), Phys. Rev. D 98, 030001 (2018).

10. T. van Ritbergen, R. G. Stuart, Nucl. Phys. B564, 343 (2000).

11. Pivovarov, A.A. Phys. Atom. Nuclei 65: 1319 (2002).

12. P. A. Baikov, K. G. Chetyrkin and J. H. Kuhn, Phys. Rev. Lett. 101, 012002 (2008).

13. A. Pich, Prog. Part. Nucl. Phys. 75, 41 (2014).

14. Bargiotti, M. et al. Riv.Nuovo Cim. 23N3, 1 (2000).

15. M. Tanabashi et al. (Particle Data Group), Phys. Rev. D 98, 030001 (2018).

16. J. Beringer et al. [Particle Data Group Collaboration], Phys. Rev. D 86, 010001 (2012).

17. F. Krinner, A. Lenz and T. Rauh, Nucl. Phys. B 876, 31 (2013).

18. Y. Fukuda et al., [Super-Kamiokande Collab.], Phys. Rev. Lett. 81, 1562 (1998).

19. Y. Ashie et al., [Super-Kamiokande Collab.], Phys. Rev. Lett. 93, 101801 (2004).

20. E. Majorana, Il Nuovo Cimento 14, 4, 171 (1937).

21. K. Wai-Yee, G. Senjanović, Phys. Rev. Lett. 50, 1427 (1983).

22. X. Qian, P. Vogel, Pro. in Part. and Nucl. Phys., 83, 1 (2015). 